\title{
Fuzzy rough sets with a fuzzy ideal
}

\author{
Ismail Ibedou ${ }^{1 *}$ (D) and S. E. Abbas ${ }^{2}$
}

\author{
*Correspondence: \\ ismail.ibedou@gmail.com; \\ sabbas73@yahoo.com \\ 'Department of Mathematics, \\ Faculty of Science, Benha University, \\ Benha, Egypt \\ Full list of author information is \\ available at the end of the article
}

\begin{abstract}
In this paper, we defined the fuzzy upper, fuzzy lower, and fuzzy boundary sets of a rough fuzzy set $\lambda$ in a fuzzy approximation space $(X, R)$. Based on $\lambda$ and $R$, we introduced the fuzzy ideal approximation interior operator intlambda ${ }^{R}$ and the fuzzy ideal approximation closure operator $\mathrm{Cl}_{\lambda}^{R}$. We joined the fuzzy ideal notion with the fuzzy approximation spaces, and then introduced the fuzzy ideal approximation closure and interior operators associated to a rough fuzzy set $\lambda$. Fuzzy ideal approximation connectedness and the fuzzy ideal approximation continuity between fuzzy ideal approximation spaces are introduced.
\end{abstract}

Keywords: Fuzzy rough set, Fuzzy ideal, Fuzzy ideal approximation space, Fuzzy ideal continuity, Fuzzy ideal connectedness

2000 Mathematics Subject Classification: 03E20, 03E72, 03E75, 54C05, 54D05

\section{Introduction}

The notion of rough sets was given by Pawlak [1] referring to uncertainty of intelligent systems characterized by insufficient and incomplete information. Basically, rough sets are defined depending on some equivalence relation $R$ on a universal finite set $X$. The pair $(X, R)$ was called an approximation space based on an equivalence relation imposed on $X$. In any approximation space, the notions of lower approximation, upper approximation, and boundary region operators of some subset could be induced. Many types of generalizations of Pawlak's rough set has been obtained by replacing equivalence relation with an arbitrary binary relation. On the other hand, the relationships between rough sets and topological spaces were studied in [2]. A lot of fuzzy generalizations of rough approximation have been proposed in the literature [3-7]. Irfan in [8] studied the connections between fuzzy set, rough set, and soft set ([9]) notions. Many papers studied the relationship between fuzzy rough set notion and fuzzy topologies [10, 11]. Recently, many researchers have used topological approaches in the study of rough sets and its applications. In [12], it was used the notion of ideal in soft rough ordinary topological space, and in [13], the authors introduced fuzzy soft connectedness in sense of Chang [14].

The motivation of this paper is to introduce a new improved fuzzy lower and fuzzy upper sets by which we get a more reliable fuzzy boundary region set of a fuzzy set $\lambda$. From these fuzzy lower and fuzzy upper sets, we can define new fuzzy interior and fuzzy closure operators associated with a specific fuzzy set $\lambda \in I^{X}$ in sense of Chang [14] and that fuzzy

(C) The Author(s). 2020 Open Access This article is licensed under a Creative Commons Attribution 4.0 International License which permits use, sharing, adaptation, distribution and reproduction in any medium or format, as long as you give appropriate credit to the original author(s) and the source, provide a link to the Creative Commons licence, and indicate if changes were made. The images or other third party material in this article are included in the article's Creative Commons licence, unless indicated otherwise in a credit line to the material. If material is not included in the article's Creative Commons licence and your intended use is not permitted by statutory regulation or exceeds the permitted use, you will need to obtain permission directly from the copyright holder. To view a copy of this licence, visit http://creativecommons.org/licenses/by/4.0/. 
relation $R$ on $X$. In the fuzzy approximation space $(X, R)$, based on these fuzzy interior and fuzzy closure operators, we defined fuzzy approximation connectedness. Defining a fuzzy ideal on $X$ generates a fuzzy ideal approximation space in which a fuzzy local function was defined and many results are proved. Connectedness in fuzzy ideal approximation spaces are defined and compared with connectedness in fuzzy approximation spaces. Also, fuzzy ideal approximation continuity between two fuzzy ideal approximation spaces were discussed. The author in [12] defined ordinary lower and upper sets but regardless to any relation on $X$. Liu in [6] introduced the fuzzy lower and fuzzy upper sets and his computations were different from our results.

Through the paper, let $X$ be a finite set of objects and $I$ the closed unit interval $[0,1]$. $I^{X}$ denotes all the fuzzy subsets of $X$, and $\lambda^{c}(x)=1-\lambda(x) \forall x \in X, \forall \lambda \in I^{X}$. A constant fuzzy set $\bar{t}$ for all $t \in I$ is defined by $\bar{t}(x)=t \forall x \in X$. Infimum and supremum of a fuzzy set $\lambda \in I^{X}$ are given as: $\inf \lambda=\bigwedge_{x \in X} \lambda(x)$ and $\sup \lambda=\bigvee_{x \in X} \lambda(x)$.

Assume a fuzzy relation $R: X \times X \rightarrow I$ is defined so that $R(x, x)=1 \forall x \in X$, $R(x, y)=R(y, x) \forall x, y \in X$ and $R(x, y) \geq(R(x, z) \wedge R(z, y)) \forall x, y, z \in X$. That is, $R$ is a fuzzy equivalence relation on $X$. $(X, R)$ is called a fuzzy approximation space based on the fuzzy equivalence relation $R$ on $X$.

Definition 1 For each $x \in X$, define a fuzzy coset $[x]: X \rightarrow I$ by:

$$
[x](y)=R(x, y) \quad \forall y \in X
$$

All elements $y \in X$ with fuzzy relation value $R(x, y)>0$ are elements having a membership value in the fuzzy coset $[x]$, and any element $y \in X$ with $R(x, y)=0$ is not included in the fuzzy coset $[x]$. Any fuzzy coset $[x]$ surely include the element $x \in X$, and consequently $\bigvee_{z \in X}([x](z))=1 \forall x \in X$. Also, $\left(\bigvee_{z \in X}[z]\right)(y)=1 \forall y \in X$ (i.e. $\left.\bigvee_{y \in X}[y]=\overline{1}\right)$. Clearly, if $R(x, y)>0$, then the fuzzy cosets $[x],[y]$ (as fuzzy sets) are containing the same elements of $X$ with some non zero membership values, and moreover, if $[y](z)=0$, then it must be that $[x](z)=0$ whenever $R(x, y)>0$. That is, any two fuzzy cosets are either two fuzzy sets containing the same elements of $X$ with some non-zero membership values or containing completely different elements of $X$ with some non-zero membership values. Strictly, in case of $I=\{0,1\}$ it is a partitioning of $X$ as usually known in the general case.

Note that $[x] \neq \overline{0} \forall x \in X$ since there is at least $x \in X$ itself such that $[x](x)=1$, while may be all elements $z \in X$ are given such that $[x](z)>0 \forall z \in X$. The fuzzy cosets could be such that $[x](x)=1$ and $[x](z)=0 \forall z \neq x$, which means $(X, R)$ is fuzzy partitioned into completely disjoint fuzzy cosets. Putting $I=\{0,1\}$ as a crisp case, we get exactly the usual meaning of partitioning of a set $X$ based on an ordinary equivalence relation $R$ on $X$.

Recall that the fuzzy difference between two fuzzy sets was defined ([15]) as:

$$
(\lambda \bar{\wedge} \mu)= \begin{cases}\overline{0} & \text { if } \lambda \leq \mu, \\ \lambda \wedge \mu^{c} & \text { otherwise. }\end{cases}
$$

\section{Fuzzy lower, fuzzy upper, and fuzzy boundary region sets}

Definition 2 Let $\lambda \in I^{X}$ and $R$ a fuzzy equivalence relation on $X$ and the fuzzy cosets are defined as in (1). Then, the fuzzy lower set $\lambda_{R}$, the fuzzy upper set $\lambda^{R}$ and the fuzzy boundary region set $\lambda^{B}$ are defined as follows: 


$$
\begin{gathered}
\lambda_{R}(x)=\lambda(x) \wedge\left(\bigvee_{\lambda^{c}(z)>0, z \neq x}[x](z)\right)^{c} \forall x \in X, \\
\lambda^{R}(x)=\lambda(x) \vee \bigvee_{\lambda(z)>0, z \neq x}[x](z) \forall x \in X, \\
\lambda^{B}=\lambda^{R} \bar{\wedge} \lambda_{R}= \begin{cases}\overline{0} & \text { if } \lambda^{R} \leq \lambda_{R} \\
\lambda^{R} \wedge\left(\lambda_{R}\right)^{c} & \text { otherwise. }\end{cases}
\end{gathered}
$$

$\lambda_{R}, \lambda^{R}$ and $\lambda^{B}$ are then called fuzzy lower, fuzzy upper, and fuzzy boundary region sets associated with the fuzzy set $\lambda$ in $I^{X}$ and based on the fuzzy equivalence relation $R$ in a fuzzy approximation space $(X, R)$.

From (3) and (4), we get that $\lambda_{R} \leq \lambda \leq \lambda^{R} \forall \lambda \in I^{X}$. Whenever $\lambda^{R}$ be so that $\lambda^{R} \leq \lambda_{R}$, we get that $\lambda=\lambda_{R}=\lambda^{R}$, and then from (5), we have $\lambda^{B}=\overline{0}$. Otherwise, $\lambda^{B}=\lambda^{R} \wedge\left(\lambda_{R}\right)^{c}$. The fuzzy accuracy $\alpha_{R}(\lambda)$ of approximation of the fuzzy set $\lambda$ could be characterized numerically by $\alpha_{R}(\lambda)=\frac{\inf \lambda_{R}}{\sup \lambda^{R}}$, where $0 \leq \alpha_{R}(\lambda) \leq 1$. If $\alpha_{R}(\lambda)=1$, then $\lambda$ is crisp with respect to $R\left(\lambda_{R}=\lambda^{R}\right.$ and $\lambda$ is precise with respect to $\left.R\right)$, and otherwise, if $\alpha_{R}(\lambda)<1, \lambda$ is rough with respect to $R$ ( $\lambda$ is vague with respect to $R$ ).

Lemma 1 For any fuzzy set $\lambda \in I^{X}$ we get that:

(1) $\lambda_{R} \leq \lambda \leq \lambda^{R}$,

(2) $\overline{0}_{R}=\overline{0}^{R}=\overline{0}$ and $\overline{1}_{R}=\overline{1}^{R}=\overline{1}$,

(3) $(\lambda \vee \mu)_{R} \geq \lambda_{R} \vee \mu_{R}$,

(4) $(\lambda \wedge \mu)^{R} \leq \lambda^{R} \wedge \mu^{R}$,

(5) $\lambda \leq \mu$ implies that $\lambda_{R} \leq \mu_{R}$ and $\lambda^{R} \leq \mu^{R}$,

(6) $(\lambda \vee \mu)^{R}=\lambda^{R} \vee \mu^{R}$,

(7) $(\lambda \wedge \mu)_{R}=\lambda_{R} \wedge \mu_{R}$,

(8) $\left(\lambda^{R}\right)^{c}=\left(\lambda^{c}\right)_{R}$ and $\left(\lambda_{R}\right)^{c}=\left(\lambda^{c}\right)^{R}$

(9) $\left(\lambda_{R}\right)^{R} \geq \lambda_{R}=\left(\lambda_{R}\right)_{R}$

(10) $\left(\lambda^{R}\right)_{R} \leq \lambda^{R}=\left(\lambda^{R}\right)^{R}$

Proof Obvious.

Remark 1 Liu in [6] has defined the fuzzy lower and the fuzzy upper sets $\underline{R} \lambda, \bar{R} \lambda: X \rightarrow I$ of a fuzzy set $\lambda \in I^{X}$ as follows:

$$
\underline{R} \lambda(x)=\bigwedge_{y \in X}\left((R(x, y))^{c} \vee \lambda(y)\right) \text { and } \bar{R} \lambda(x)=\bigvee_{y \in X}(R(x, y) \wedge \lambda(y)) \forall x \in X .
$$

In the following example, we will see that the fuzzy lower and the fuzzy upper sets of some fuzzy set will be different if we used the above notations and our definitions.

Example 1 Let $R$ be a fuzzy relation on a set $X=\{a, b, c, d\}$ as shown down.

\begin{tabular}{|c||c|c|c|c}
\hline$R$ & $a$ & $b$ & $c$ & $d$ \\
\hline \hline$a$ & 1 & 0.8 & 0.3 & 0.1 \\
\hline$b$ & 0.8 & 1 & 0.3 & 0.1 \\
\hline$c$ & 0.3 & 0.3 & 1 & 0.1 \\
\hline$d$ & 0.1 & 0.1 & 0.1 & 1 \\
\hline
\end{tabular}


Assume that $\lambda=\{0.3,0.4,1,0.2\}$. Then, the fuzzy cosets are as follows:

$$
\begin{aligned}
& {[a]=\{1,0.8,0.3,0.1\}, \underset{\lambda(z)>0, z \neq a}{\bigvee}[a](z)=0.8,\left(\underset{\lambda^{c}(z)>0, z \neq a}{\bigvee}[a](z)\right)^{c}=0.2} \\
& {[b]=\{0.8,1,0.3,0.1\}, \bigvee_{\lambda(z)>0, z \neq b}[b](z)=0.8,\left(\bigvee_{\lambda^{c}(z)>0, z \neq b}[b](z)\right)^{c}=0.2} \\
& {[c]=\{0.3,0.3,1,0.1\}, \quad \bigvee_{\lambda(z)>0, z \neq c}[c](z)=0.3, \quad\left(\bigvee_{\lambda^{c}(z)>0, z \neq c}[c](z)\right)^{c}=0.7} \\
& {[d]=\{0.1,0.1,0.1,1\}, \quad \bigvee_{\lambda(z)>0, z \neq d}[d](z)=0.1,\left(\bigvee_{\lambda^{c}(z)>0, z \neq d}[d](z)\right)^{c}=0.9 \text {. }}
\end{aligned}
$$

Now, $\lambda_{R}=\{0.2,0.2,0.7,0.2\}, \lambda^{R}=\{0.8,0.8,1,0.2\}$ and then

$\lambda^{B}=\{0.8,0.8,0.3,0.2\}$. But $\underline{R} \lambda=\{0.3,0.3,0.7,0.2\}$ and $\bar{R} \lambda=\{0.4,0.4,1,0.2\}$ are different from our fuzzy approximation sets.

$\lambda^{c}=\{0.7,0.6,0,0.8\}$ implies that $\left(\lambda^{c}\right)_{R}=\{0.2,0.2,0,0.8\}$ equal to $\left(\lambda^{R}\right)^{c}$ and $\left(\lambda^{c}\right)^{R}=\{0.8,0.8,0.3,0.8\}$ equal to $\left(\lambda_{R}\right)^{c}$.

For $\mu=\{0.6,0.2,0,1\}$, we get that $\mu_{R}=\{0.2,0.2,0,0.9\}, \mu^{R}=\{0.8,0.8,0.3,1\}$ and then $\mu^{B}=\{0.8,0.8,0.3,0.1\}$.

Here, $(\lambda \wedge \mu)=\{0.3,0.2,0,0.2\}$, and then $(\lambda \wedge \mu)_{R}=\{0.2,0.2,0,0.2\}$ equal to $\lambda_{R} \wedge \mu_{R}$ and $(\lambda \vee \mu)^{R}=\{0.6,0.4,1,1\}^{R}=\{0.8,0.8,1,1\}$ equal to $\lambda^{R} \vee \mu^{R}$, moreover $(\lambda \wedge \mu)^{R}=$ $\{0.8,0.8,0.3,0.2\}$ equal to $\lambda^{R} \wedge \mu^{R}$ and $(\lambda \vee \mu)_{R}=\{0.2,0.2,0.7,0.9\}$ equal to $\lambda_{R} \vee \mu_{R}$.

Example 2 Let $R$ be a fuzzy relation on a set $X=\{a, b, c, d\}$ as shown in the matrix:

\begin{tabular}{|c||c|c|c|c|}
\hline$R$ & $a$ & $b$ & $c$ & $d$ \\
\hline \hline$a$ & 1 & 0.4 & 0.2 & 0.2 \\
\hline$b$ & 0.4 & 1 & 0.2 & 0.2 \\
\hline$c$ & 0.2 & 0.2 & 1 & 0.2 \\
\hline$d$ & 0.2 & 0.2 & 0.2 & 1 \\
\hline
\end{tabular}

Assume that $\lambda=\overline{0.5}$. Then, the fuzzy cosets are as follows:

$$
\begin{aligned}
& \underset{\lambda(z)>0, z \neq a}{\bigvee}[a](z)=0.4, \quad \underset{\lambda(z)>0, z \neq b}{\bigvee}[b](z)=0.4, \quad \underset{\lambda(z)>0, z \neq c}{\bigvee}[c](z)=0.2, \\
& \underset{\lambda(z)>0, z \neq d}{\bigvee}[d](z)=0.2, \\
& \left(\underset{\lambda^{c}(z)>0, z \neq a}{\bigvee}[a](z)\right)^{c}=0.6,\left(\underset{\lambda^{c}(z)>0, z \neq b}{\bigvee}[b](z)\right)^{c}=0.6, \\
& \left(\underset{\lambda^{c}(z)>0, z \neq c}{\bigvee}[c](z)\right)^{c}=0.8,\left(\underset{\lambda^{c}(z)>0, z \neq d}{\bigvee}[d](z)\right)^{c}=0.8 .
\end{aligned}
$$

Here, $\lambda_{R}=\lambda^{R}=\lambda=\overline{0.5}$ and then $\lambda^{B}=\overline{0}$.

For $\mu=\{0.6,0.7,0.8,0.9\}$, we get that $\mu^{R}=\{0.6,0.7,0.8,0.9\}=\mu$

but $\mu_{R}=\{0.6,0.6,0.8,0.8\} \neq \mu$, and then $\mu^{B}=\{0.4,0.4,0.2,0.2\}$.

Associated with a fuzzy set $\lambda$ in a fuzzy approximation space $(X, R)$, we can define a fuzzy interior operator $\operatorname{int}_{R}^{\lambda}: I^{X} \rightarrow I^{X}$ as follows:

$$
\operatorname{int}_{R}^{\lambda}(v)=\lambda_{R} \wedge v_{R} \forall v \neq \overline{1} \text { and } \operatorname{int}_{R}^{\lambda}(\overline{1})=\overline{1} \text {. }
$$

Lemma 2 The following conditions are satisfied.

(1) $\operatorname{int}_{R}^{\lambda}(\overline{0})=\overline{0}$, 
(2) $\operatorname{int}_{R}^{\lambda}(v) \leq v \forall v \in I^{X}$,

(3) $v \leq \eta \Longrightarrow \operatorname{int} t_{R}^{\lambda}(v) \leq \operatorname{int} t_{R}^{\lambda}(\eta) \forall v, \eta \in I^{X}$,

(4) $\operatorname{int}_{R}^{\lambda}(v \wedge \eta)=\operatorname{int} t_{R}^{\lambda}(v) \wedge \operatorname{int} t_{R}^{\lambda}(\eta), \operatorname{int} t_{R}^{\lambda}(v \vee \eta)=\operatorname{int} t_{R}^{\lambda}(v) \vee \operatorname{int} t_{R}^{\lambda}(\eta) \forall v, \eta \in I^{X}$,

(5) $\operatorname{int}_{R}^{\lambda}\left(\operatorname{int}_{R}^{\lambda}(v)\right)=\operatorname{int}_{R}^{\lambda}(v) \forall v \in I^{X}$.

Proof For (1): $\operatorname{int}_{R}^{\lambda}(\overline{0})=\lambda_{R} \wedge(\overline{0})_{R}=\overline{0}$.

For (2): $\operatorname{int}_{R}^{\lambda}(v)=\lambda_{R} \wedge v_{R} \leq \lambda_{R} \wedge v \leq v$.

For (3): If $v \leq \eta$ then $v_{R} \leq \eta_{R} \Longrightarrow \operatorname{int}_{R}^{\lambda}(v) \leq \operatorname{int}_{R}^{\lambda}(\eta)$.

For (4): $\operatorname{int}_{R}^{\lambda}(\nu \wedge \eta)=\lambda_{R} \wedge(v \wedge \eta)_{R}=\lambda_{R} \wedge\left(v_{R} \wedge \eta_{R}\right)=\left(\lambda_{R} \wedge v_{R}\right) \wedge\left(\lambda_{R} \wedge \eta_{R}\right)=$ $\operatorname{int}_{R}^{\lambda}(\nu) \wedge \operatorname{int}_{R}^{\lambda}(\eta)$

For (5): $\operatorname{int}_{R}^{\lambda}\left(\operatorname{int}_{R}^{\lambda}(v)\right)=\lambda_{R} \wedge\left(\operatorname{int}_{R}^{\lambda}(v)\right)_{R}=\lambda_{R} \wedge\left(\lambda_{R} \wedge v_{R}\right)_{R}=\lambda_{R} \wedge\left(\lambda_{R}\right)_{R} \wedge\left(v_{R}\right)_{R}=$ $\lambda_{R} \wedge v_{R}=\operatorname{int}_{R}^{\lambda}(v)$.

Thus, this is called a fuzzy interior associated with $\lambda$ in the fuzzy approximation space $(X, R)$ generating a fuzzy topology defined by:

$$
\varpi_{R}^{\lambda}=\left\{v \in I^{X}: v=\operatorname{int}_{R}^{\lambda}(v)\right\} .
$$

Also, we can define a fuzzy closure operator $\mathrm{cl}_{R}^{\lambda}: I^{X} \rightarrow I^{X}$ as follows:

$$
\mathrm{cl}_{R}^{\lambda}(v)=\left(\lambda_{R}\right)^{c} \vee v^{R} \forall v \neq \overline{0} \quad \text { and } \mathrm{cl}_{R}^{\lambda}(\overline{0})=\overline{0} .
$$

Note that:

$$
\begin{aligned}
& \operatorname{cl}_{R}^{\lambda}\left(v^{R}\right)=\operatorname{cl}_{R}^{\lambda}(v) \forall v \in I^{X}, \quad \operatorname{int}_{R}^{\lambda}\left(v_{R}\right)=\operatorname{int}_{R}^{\lambda}(v) \forall v \in I^{X}, \\
& \left.\operatorname{int}_{R}^{\lambda}\left(v^{c}\right)=\left(\operatorname{cl}_{R}^{\lambda}(v)\right)^{c} \text { and } \operatorname{cl}_{R}^{\lambda}\left(v^{c}\right)=\operatorname{int}_{R}^{\lambda}(v)\right)^{c} \forall v \in I^{X} .
\end{aligned}
$$

Lemma 3 The fuzzy closure operator satisfy the following conditions:

(1) $c l_{R}^{\lambda}(\overline{1})=\overline{1}$,

(2) $c l_{R}^{\lambda}(v) \geq v \forall v \in I^{X}$,

(3) $v \leq \eta \Longrightarrow c l_{R}^{\lambda}(v) \leq c l_{R}^{\lambda}(\eta) \forall v, \eta \in I^{X}$,

(4) $c l_{R}^{\lambda}(v \wedge \eta)=c l_{R}^{\lambda}(v) \wedge c l_{R}^{\lambda}(\eta), c l_{R}^{\lambda}(v \vee \eta)=c l_{R}^{\lambda}(v) \vee c l_{R}^{\lambda}(\eta) \forall v, \eta \in I^{X}$,

(5) $c l_{R}^{\lambda}\left(c l_{R}^{\lambda}(v)\right)=c l_{R}^{\lambda}(v) \forall v \in I^{X}$.

Proof similar to Lemma 2.

Hence, from $\mathrm{cl}_{R}^{\lambda}\left(v^{c}\right)=\left(\operatorname{int}_{R}^{\lambda}(v)\right)^{c}$, it is a fuzzy closure operator generating the same fuzzy topology given above (from (8) in Lemma 1) as follows:

$$
\varpi_{R}^{\lambda}=\left\{v \in I^{X}: v^{c}=\operatorname{cl}_{R}^{\lambda}\left(v^{c}\right)\right\} .
$$

\section{Fuzzy ideal approximation spaces}

A subset $\ell \subset I^{X}$ is called a fuzzy ideal ([16]) on $X$ if it satisfies the following conditions:

(1) $\overline{0} \in \ell$,

(2) If $v \leq \mu$ and $\mu \in \ell$, then $v \in \ell$ for all $\mu, v \in I^{X}$,

(3) If $\mu \in \ell$ and $v \in \ell$, then $(\mu \vee v) \in \ell$ for all $\mu, v \in I^{X}$. 
If $\ell_{1}$ and $\ell_{2}$ are fuzzy ideals on $X$, we have $\ell_{1}$ is finer than $\ell_{2}\left(\ell_{2}\right.$ is coarser than $\left.\ell_{1}\right)$ if $\ell_{1} \supseteq \ell_{2}$. The triple $(X, R, \ell)$ is called a fuzzy ideal approximation space. Denote the trivial fuzzy ideal $\ell^{\circ}$ as a fuzzy ideal including only $\overline{0}$.

Definition 3 Let $(X, R, \ell)$ be a fuzzy ideal approximation space and $\lambda \in I^{X}$. Then, the fuzzy local set $\mu_{\lambda}^{*}(R, \ell)$ of a set $\mu \in I^{X}$ is defined by:

$$
\mu_{\lambda}^{*}(R, \ell)=\bigwedge\left\{v \in I^{X}:(\mu \bar{\wedge} \nu) \in \ell, c l_{R}^{\lambda}(\nu)=v\right\} .
$$

For short, we will write $\mu_{\lambda}^{*}$ or $\mu_{\lambda}^{*}(\ell)$ instead of $\mu_{\lambda}^{*}(R, \ell)$.

Corollary 1 Let $\left(X, R, \ell^{\circ}\right)$ be a fuzzy ideal approximation space, $\lambda \in I^{X}$ where $\ell^{\circ}$ is the trivial fuzzy ideal on $X$. Then, for each $\mu \in I^{X}$, we have $\mu_{\lambda}^{*}=c l_{R}^{\lambda}(\mu)$.

Proof Since $\ell^{\circ}=\{\overline{0}\}$, we get that $\mu_{\lambda}^{*}=\bigwedge\left\{v \in I^{X}:(\mu \bar{\wedge} v)=\overline{0}, \mathrm{cl}_{R}^{\lambda}(\nu)=v\right\}$, that is, $\mu_{\lambda}^{*}=\bigwedge\left\{v \in I^{X}: \mu \leq v, \operatorname{cl}_{R}^{\lambda}(v)=v\right\}$. Since $\mu \leq \mathrm{cl}_{R}^{\lambda}(\mu), \mathrm{cl}_{R}^{\lambda}\left(\mathrm{cl}_{R}^{\lambda}(\mu)\right)=\mathrm{cl}_{R}^{\lambda}(\mu)$, then $\mu_{\lambda}^{*} \leq \operatorname{cl}_{R}^{\lambda}(\mu)$. Suppose that $\operatorname{cl}_{R}^{\lambda}(\mu) \not \mu_{\lambda}^{*}$, then there exists $v \in I^{X}, \mu \leq v, \operatorname{cl}_{R}^{\lambda}(\nu)=v$ so that $\operatorname{cl}_{R}^{\lambda}(\mu)>v$. But $\mu \leq v$ implies that $\mu^{R} \leq v^{R}$, and then

$$
\mathrm{cl}_{R}^{\lambda}(\mu)=\left(\lambda_{R}\right)^{c} \vee \mu^{R} \leq\left(\lambda_{R}\right)^{c} \vee v^{R}=\mathrm{cl}_{R}^{\lambda}(\nu)=\nu .
$$

Contradiction, and then $\mu_{\lambda}^{*}=\mathrm{cl}_{R}^{\lambda}(\mu)$.

Example 3 This is an example showing that $\mu_{\lambda}^{*} \leq c l_{R}^{\lambda}(\mu) \forall \mu \in I^{X}$. Let $R$ be a fuzzy relation on a set $X=\{a, b, c, d, e\}$ as shown down.

\begin{tabular}{|c||c|c|c|c|c|}
\hline$R$ & $a$ & $b$ & $c$ & $d$ & $e$ \\
\hline \hline$a$ & 1 & 0 & 0 & 0 & 0 \\
\hline$b$ & 0 & 1 & 0 & 0 & 0 \\
\hline$c$ & 0 & 0 & 1 & 0 & 0 \\
\hline$d$ & 0 & 0 & 0 & 1 & 0.2 \\
\hline$e$ & 0 & 0 & 0 & 0.2 & 1 \\
\hline
\end{tabular}

Assume that $\lambda=\{1,1,1,0.1,0.2\}$. Then,

$$
\begin{gathered}
\bigvee_{\lambda(z)>0, z \neq x}[x](z)=0 \forall x \in\{a, b, c\}, \underset{\lambda(z)>0, z \neq x}{\bigvee}[x](z)=0.2 \forall x \in\{d, e\}, \\
\left(\underset{\lambda^{c}(z)>0, z \neq x}{\bigvee}[x](z)\right)^{c}=1 \forall x \in\{a, b, c\} \text { and }\left(\bigvee_{\lambda^{c}(z)>0, z \neq x}[x](z)\right)^{c}=0.8 \forall x \in\{d, e\} .
\end{gathered}
$$

Hence, $\lambda_{R}=\{1,1,1,0.1,0.2\}$ and $\lambda^{R}=\{1,1,1,0.2,0.2\}$, and $\left(\lambda_{R}\right)^{c}=\{0,0,0,0.9,0.8\}$. Let $\mu=\{0.3,0,0,0.8,0.8\}$, then $\mu^{R}=\mu=\{0.3,0,0,0.8,0.8\}$, and thus $\left(\mu^{R} \vee\left(\lambda_{R}\right)^{c}\right)=\mathrm{cl}_{R}^{\lambda}(\mu)=\{0.3,0,0,0.9,0.8\}$.

Assume that a fuzzy ideal is defined on $X$ as follows $\ell=\left\{\eta \in I^{X}: \eta \leq \overline{0.6}\right\}$.

Note that for any $\eta \in I^{X}$, we have $c l_{R}^{\lambda}(\eta) \geq\{0,0,0,0.9,0.8\}$ and recall that if $\mu \in \ell$, then $\mu_{\lambda}^{*}=\overline{0}$ direct (because $\mu \bar{\wedge} \overline{0}=\mu \wedge \overline{1}=\mu \in \ell$ and $c l_{R}^{\lambda}(\overline{0})=\overline{0}$ ), and if $c l_{R}^{\lambda}(\mu)=\mu$ and $\mu \notin \ell$, then $\mu_{\lambda}^{*}=\mu$.

Now, we get that for any choice $\eta=\{p, 0,0,0.9,0.8\}$ for all $p \in I$, we get $c l_{R}^{\lambda}(\eta)=\eta$ and $\mu \bar{\wedge} \eta \in \ell$, and thus $\mu_{\lambda}^{*}=\{0,0,0,0.9,0.8\} \nsucceq c l_{R}^{\lambda}(\mu)$.

If we defined the fuzzy ideal as follows $\ell=\left\{\eta \in I^{X}: \eta \leq \overline{0.2}\right\}$.

Then, for any $\eta=\{p, 0,0,0.9,0.8\}$ with $p<0.3$, we get that $c l_{R}^{\lambda}(\eta)=\eta$ but $\mu \bar{\wedge} \eta=\{0.3,0,0,0.1,0.2\} \notin \ell$, and thus $\mu_{\lambda}^{*}=\{0.3,0,0,0.9,0.8\}=\operatorname{cl}_{R}^{\lambda}(\mu)$. Hence, $\mu_{\lambda}^{*} \leq \mathrm{cl}_{R}^{\lambda}(\mu)$ in general. 
Proposition 1 Let $(X, R, \ell)$ be a fuzzy ideal approximation space and $\lambda \in I^{X}$. Then,

(1) $\mu \leq v$ implies $\mu_{\lambda}^{*} \leq v_{\lambda}^{*}$.

(2) If $\ell_{1}, \ell_{2}$ are fuzzy ideals on $X$ and $\ell_{1} \subseteq \ell_{2}$, then $\mu_{\lambda}^{*}\left(\ell_{1}\right) \geq \mu_{\lambda}^{*}\left(\ell_{2}\right)$.

(3) $\operatorname{int}_{R}^{\lambda}\left(\mu_{\lambda}^{*}\right) \leq \mu_{\lambda}^{*}=\operatorname{cl}_{R}^{\lambda}\left(\mu_{\lambda}^{*}\right) \leq c l_{R}^{\lambda}(\mu)$.

(4) $\left(\mu_{\lambda}^{*}\right)_{\lambda}^{*} \leq c l_{R}^{\lambda}\left(\mu_{\lambda}^{*}\right)$.

(5) $\mu_{\lambda}^{*} \vee v_{\lambda}^{*} \leq(\mu \vee v)_{\lambda}^{*}$ and $\mu_{\lambda}^{*} \wedge v_{\lambda}^{*} \geq(\mu \wedge v)_{\lambda}^{*}$.

Proof (1): Suppose that $\mu_{\lambda}^{*} \not \leq \nu_{\lambda}^{*}$, then there exists $\omega \in I^{X}$ with $(\nu \bar{\wedge} \omega) \in \ell$ and $\mathrm{cl}_{R}^{\lambda}(\omega)=$ $\omega$ such that $\mu_{\lambda}^{*}>\omega \geq v_{\lambda}^{*}$. Since $\mu \leq v$, then $\mu \bar{\wedge} \omega \leq \nu \bar{\wedge} \omega$ and $(\mu \bar{\wedge} \omega) \in \ell, \operatorname{cl}_{R}^{\lambda}(\omega)=\omega$. Thus, $\mu_{\lambda}^{*} \leq \omega$, which is a contradiction and hence $\mu_{\lambda}^{*} \leq v_{\lambda}^{*}$.

(2): Suppose that $\mu_{\lambda}^{*}\left(\ell_{1}\right) \geq \mu_{\lambda}^{*}\left(\ell_{2}\right)$, then there exists $\omega \in I^{X}$ with $(\mu \bar{\wedge} \omega) \in \ell_{1}$ and $\operatorname{cl}_{R}^{\lambda}(\omega)=\omega$ such that $\mu_{\lambda}^{*}\left(\ell_{1}\right) \leq \omega<\mu_{\lambda}^{*}\left(\ell_{2}\right)$. Since $\ell_{1} \subseteq \ell_{2}$, then $(\mu \bar{\wedge} \omega) \in \ell_{2}$ and $\mathrm{cl}_{R}^{\lambda}(\omega)=\omega$, and then $\mu_{\lambda}^{*}\left(\ell_{2}\right) \leq \omega$, which is a contradiction. Thus, $\mu_{\lambda}^{*}\left(\ell_{1}\right) \geq \mu_{\lambda}^{*}\left(\ell_{2}\right)$.

(3): $\operatorname{int}_{R}^{\lambda}\left(\mu_{\lambda}^{*}\right) \leq \mu_{\lambda}^{*} \leq \operatorname{cl}_{R}^{\lambda}\left(\mu_{\lambda}^{*}\right)$ direct. Since $\mu_{\lambda}^{*} \leq \operatorname{cl}_{R}^{\lambda}(\mu)$, then $\operatorname{cl}_{R}^{\lambda}\left(\mu_{\lambda}^{*}\right) \leq \mathrm{cl}_{R}^{\lambda}(\mu)$.

(4): Since $\mu_{\lambda}^{*} \leq \mathrm{cl}_{R}^{\lambda}(\mu) \forall \mu \in I^{X}$, then $\left(\mu_{\lambda}^{*}\right)_{\lambda}^{*} \leq \operatorname{cl}_{R}^{\lambda}\left(\mu_{\lambda}^{*}\right)$.

(5): From (1), we have $\mu \leq v \Longrightarrow \mu_{\lambda}^{*} \leq v_{\lambda}^{*}$, and so (5) is satisfied.

Definition 4 Let $(X, R, \ell)$ be a fuzzy ideal approximation space and $\lambda \in I^{X}$. Then,

$$
\begin{aligned}
& \left(c l_{R}^{\lambda}\right)_{\lambda}^{*}(\mu)=c l_{R}^{\lambda}(\mu) \vee\left(\lambda^{R}\right)_{\lambda}^{*} \quad \forall \mu \in I^{X} . \\
& \left(\operatorname{int} t_{R}^{\lambda}\right)_{\lambda}^{*}(\mu)=\operatorname{int} t_{R}^{\lambda}(\mu) \wedge\left(\left(\lambda^{R}\right)_{\lambda}^{*}\right)^{c} \quad \forall \mu \in I^{X} .
\end{aligned}
$$

$\left(c l_{R}^{\lambda}\right)_{\lambda}^{*}$ and $\left(i n t_{R}^{\lambda}\right)_{\lambda}^{*}$ are fuzzy operators from $I^{X}$ into $I^{X}$ based on a specific fuzzy set $\lambda$ and a fuzzy ideal $\ell$ in the fuzzy approximation space $(X, R)$.

Now, if $\ell=\ell^{\circ}$, then from Equation 2.7, Corollary 1, Lemma 2 and Lemma 3,

$$
\begin{aligned}
& \left(\mathrm{cl}_{R}^{\lambda}\right)_{\lambda}^{*}(\mu)=\operatorname{cl}_{R}^{\lambda}(\mu \vee \lambda) \geq \operatorname{cl}_{R}^{\lambda}(\mu)=\operatorname{cl}_{R}^{\lambda}\left(\mu_{\lambda}^{*}\right)=\mu_{\lambda}^{*} \text { and, } \\
& \left(\operatorname{int}_{R}^{\lambda}\right)_{\lambda}^{*}(\mu)=\operatorname{int}_{R}^{\lambda}\left(\mu \wedge \lambda^{c}\right) \leq \operatorname{int}_{R}^{\lambda}(\mu)=\operatorname{int}_{R}^{\lambda}\left(\left(\left(\mu^{c}\right)_{\lambda}^{*}\right)^{c}\right)=\left(\left(\mu^{c}\right)_{\lambda}^{*}\right)^{c} \forall \mu \in I^{X} .
\end{aligned}
$$

Proposition 2 Let $(X, R, \ell)$ be a fuzzy ideal approximation space with $\lambda \in I^{X}$ fixed. Then, for any $\mu, v \in I^{X}$, we have:

(1) $\left(\operatorname{int}_{R}^{\lambda}\right)_{\lambda}^{*}(\mu) \leq \operatorname{int}_{R}^{\lambda}(\mu) \leq \mu \leq c l_{R}^{\lambda}(\mu) \leq\left(c l_{R}^{\lambda}\right)_{\lambda}^{*}(\mu)$.

(2) $\left(c l_{R}^{\lambda}\right)_{\lambda}^{*}\left(\mu^{c}\right)=\left(\left(\text { int }_{R}^{\lambda}\right)_{\lambda}^{*}(\mu)\right)^{c}$ and $\left(\text { int }_{R}^{\lambda}\right)_{\lambda}^{*}\left(\mu^{c}\right)=\left(\left(c l_{R}^{\lambda}\right)_{\lambda}^{*}(\mu)\right)^{c}$.

(3) $\left(c l_{R}^{\lambda}\right)_{\lambda}^{*}(\mu \vee v)=\left(c l_{R}^{\lambda}\right)_{\lambda}^{*}(\mu) \vee\left(\mathrm{cl}_{R}^{\lambda}\right)_{\lambda}^{*}(v)$ and $\left(\operatorname{int}_{R}^{\lambda}\right)_{\lambda}^{*}(\mu \wedge v)=\left(\operatorname{int}_{R}^{\lambda}\right)_{\lambda}^{*}(\mu) \wedge\left(\operatorname{int}_{R}^{\lambda}\right)_{\lambda}^{*}(\nu)$.

(4) $\left(c l_{R}^{\lambda}\right)_{\lambda}^{*}(\mu \wedge v)=\left(c l_{R}^{\lambda}\right)_{\lambda}^{*}(\mu) \wedge\left(\mathrm{cl}_{R}^{\lambda}\right)_{\lambda}^{*}(v)$ and $\left(\operatorname{int}_{R}^{\lambda}\right)_{\lambda}^{*}(\mu \vee v)=\left(\operatorname{int}_{R}^{\lambda}\right)_{\lambda}^{*}(\mu) \vee\left(\operatorname{int}_{R}^{\lambda}\right)_{\lambda}^{*}(v)$.

(5) $\left(c l_{R}^{\lambda}\right)_{\lambda}^{*}\left(\left(c l_{R}^{\lambda}\right)_{\lambda}^{*}(\mu)\right) \geq\left(c l_{R}^{\lambda}\right)_{\lambda}^{*}(\mu)$ and $\left.(\text { int })_{R}^{\lambda}\right)_{\lambda}^{*}\left(\left(\text { int }{ }_{R}^{\lambda}\right)_{\lambda}^{*}(\mu)\right) \leq\left(\text { int } t_{R}^{\lambda}\right)_{\lambda}^{*}(\mu)$.

(6) If $\mu \leq v$, then $\left(c l_{R}^{\lambda}\right)_{\lambda}^{*}(\mu) \leq\left(c l_{R}^{\lambda}\right)_{\lambda}^{*}(v)$ and $\left(\text { int } t_{R}^{\lambda}\right)_{\lambda}^{*}(\mu) \leq\left(\text { int } t_{R}^{\lambda}\right)_{\lambda}^{*}(v)$. 
(2): From (8) in Lemma 1, we get that

$$
\begin{aligned}
{\left[\left(\operatorname{int}_{R}^{\lambda}\right)_{\lambda}^{*}(\mu)\right]^{c} } & =\left[\operatorname{int}_{R}^{\lambda}(\mu) \wedge\left(\left(\lambda^{R}\right)_{\lambda}^{*}\right)^{c}\right]^{c} \\
& =\left[\operatorname{int}_{R}^{\lambda}(\mu)\right]^{c} \vee\left(\lambda^{R}\right)_{\lambda}^{*} \\
& =\operatorname{cl}_{R}^{\lambda}\left(\mu^{c}\right) \vee\left(\lambda^{R}\right)_{\lambda}^{*} \\
& =\left(\operatorname{cl}_{R}^{\lambda}\right)_{\lambda}^{*}\left(\mu^{c}\right) .
\end{aligned}
$$

By the same way, you can prove that $\left(\operatorname{int}_{R}^{\lambda}\right)_{\lambda}^{*}\left(\mu^{c}\right)=\left(\left(\mathrm{cl}_{R}^{\lambda}\right)_{\lambda}^{*}(\mu)\right)^{c}$.

(3) - (4): From Equations 3.2, 3.3, (4) in Lemma 2.2 and (4) in Lemma 2.3, we get the proof.

(5): $\left(\mathrm{cl}_{R}^{\lambda}\right)_{\lambda}^{*}\left(\left(\mathrm{cl}_{R}^{\lambda}\right)_{\lambda}^{*}(\mu)\right)=\operatorname{cl}_{R}^{\lambda}\left(\left(\mathrm{cl}_{R}^{\lambda}\right)_{\lambda}^{*}(\mu)\right) \vee\left(\lambda^{R}\right)_{\lambda}^{*} \geq\left(\mathrm{cl}_{R}^{\lambda}\right)_{\lambda}^{*}(\mu) \vee\left(\lambda^{R}\right)_{\lambda}^{*} \geq\left(\mathrm{cl}_{R}^{\lambda}\right)_{\lambda}^{*}(\mu)$.

(6): From (3) in Lemma 2.3, we get $\mu \leq v \Rightarrow \mathrm{cl}_{R}^{\lambda}(\mu) \leq \mathrm{cl}_{R}^{\lambda}(v)$, and then

$\left(\operatorname{cl}_{R}^{\lambda}\right)_{\lambda}^{*}(\mu) \leq\left(\operatorname{cl}_{R}^{\lambda}\right)_{\lambda}^{*}(v)$. Similarly, $\left(\operatorname{int}_{R}^{\lambda}\right)_{\lambda}^{*}(\mu) \leq\left(\operatorname{int}_{R}^{\lambda}\right)_{\lambda}^{*}(v)$.

\section{Connectedness in fuzzy ideal approximation spaces}

Definition 5 Let $(X, R)$ be a fuzzy approximation space and $\lambda \in I^{X}$. Then,

(1) The fuzzy sets $\mu, v \in I^{X}$ are called fuzzy approximation separated if

$$
c l_{R}^{\lambda}(\mu) \wedge \nu=\mu \wedge c l_{R}^{\lambda}(v)=\overline{0} .
$$

(2) A fuzzy set $\eta \in I^{X}$ is called fuzzy approximation disconnected set if there exist fuzzy approximation separated sets $\mu, v \in I^{X}$, such that $\mu \vee v=\eta$. A fuzzy set $\eta$ is called fuzzy approximation connected (FA -connected) if it is not fuzzy approximation disconnected.

(3) $(X, R)$ is called fuzzy approximation disconnected space if there exist fuzzy approximation separated sets $\mu, v \in I^{X}$, such that $\mu \vee v=\overline{1}$. A fuzzy approximation space $(X, R)$ is called fuzzy approximation connected (FA-connected) if it is not fuzzy approximation disconnected.

Definition 6 Let $(X, R, \ell)$ be a fuzzy ideal approximation space and $\lambda \in I^{X}$. Then,

(1) The fuzzy sets $\mu, v \in I^{X}$ are called fuzzy ideal approximation separated if

$$
\left(c l_{R}^{\lambda}\right)_{\lambda}^{*}(\mu) \wedge v=\mu \wedge\left(c l_{R}^{\lambda}\right)_{\lambda}^{*}(v)=\overline{0}
$$

(2) A fuzzy set $\eta \in I^{X}$ is called fuzzy ideal approximation disconnected set if there exist fuzzy ideal approximation separated sets $\mu, v \in I^{X}$, such that $\mu \vee v=\eta$. A fuzzy set $\eta$ is called fuzzy ideal approximation connected (FIA -connected) if it is not fuzzy ideal approximation disconnected.

(3) $(X, R, \ell)$ is called fuzzy ideal approximation disconnected space if there exist fuzzy ideal approximation separated sets $\mu, v \in I^{X}$, such that $\mu \vee v=\overline{1}$. A fuzzy ideal approximation space $(X, R, \ell)$ is called fuzzy ideal approximation connected (FIA -connected) if it is not fuzzy ideal approximation disconnected.

Remark 2 Any two fuzzy ideal approximation separated sets $\mu$, $v$ in $I^{X}$ are fuzzy approximation separated as well (from that $c l_{R}^{\lambda}(\omega) \leq\left(c l_{R}^{\lambda}\right)_{\lambda}^{*}(\omega) \forall \omega \in I^{X}$ ). That is, fuzzy ideal approximation disconnectedness implies fuzzy approximation disconnectedness and thus, fuzzy approximation connectedness implies fuzzy ideal approximation connectedness. 
Example 4 Let $X=\{a, b, c, d, e\}, R$ a fuzzy relation defined by

\begin{tabular}{|c||c|c|c|c|c|}
\hline$R$ & $a$ & $b$ & $c$ & $d$ & $e$ \\
\hline \hline$a$ & 1 & 0.1 & 0 & 0 & 0 \\
\hline$b$ & 0.1 & 1 & 0 & 0 & 0 \\
\hline$c$ & 0 & 0 & 1 & 0 & 0 \\
\hline$d$ & 0 & 0 & 0 & 1 & 0 \\
\hline$e$ & 0 & 0 & 0 & 0 & 1 \\
\hline
\end{tabular}

Suppose that $\lambda=\{0,0,0.2,1,1\}$. Then, $\bigvee_{\lambda(z)>0, z \neq x}[x](z)=0 \forall x \in X$, $\left(\bigvee_{\lambda^{c}(z)>0, z \neq x}[x](z)\right)^{c}=0.9 \forall x \in\{a, b\}$ and $\left(\bigvee_{\lambda^{c}(z)>0, z \neq x}[x](z)\right)^{c}=1 \forall x \in\{c, d, e\}$.

Hence, $\lambda_{R}=\{0,0,0.2,1,1\}=\lambda^{R}$, and $\left(\lambda_{R}\right)^{c}=\{1,1,0.8,0,0\}$.

Let $\mu=\{0,0,0,0.6,0\}, v=\{0,0,0,0,0.6\}$. Then, $\mu^{R}=\{0,0,0,0.6,0\}$, $v^{R}=\{0,0,0,0,0.6\}$, and then $\left(\mu^{R} \vee\left(\lambda_{R}\right)^{c}\right)=c l_{R}^{\lambda}(\mu)=\{1,1,0.8,0.6,0\}$ and $\left(v^{R} \vee\left(\lambda_{R}\right)^{c}\right)=c l_{R}^{\lambda}(v)=\{1,1,0.8,0,0.6\}$, which means that

$$
c l_{R}^{\lambda}(\mu) \wedge v=c l_{R}^{\lambda}(v) \wedge \mu=\overline{0} .
$$

Thus, $\mu, v$ are fuzzy approximation separated sets, and moreover the fuzzy set $(\mu \vee v)=$ $\{0,0,0,0.6,0.6\}$ is fuzzy approximation disconnected set.

Now, consider the fuzzy ideal $\ell$ is defined by $\ell=\left\{\eta \in I^{X}: \quad \eta \leq \overline{0.7}\right\}$. Then, $\lambda^{R}=\{0,0,0.2,1,1\}$ implies that $\left(\lambda^{R}\right)_{\lambda}^{*}=\{1,1,0.8,0.3,0.3\}$, and then

$$
\left(c l_{R}^{\lambda}\right)_{\lambda}^{*}(\mu)=c l_{R}^{\lambda}(\mu) \vee\left(\lambda^{R}\right)_{\lambda}^{*}=\{1,1,0.8,0.6,0.3\},
$$

which means that $\left(c l_{R}^{\lambda}\right)_{\lambda}^{*}(\mu) \wedge v=\{0,0,0,0,0.3\} \neq \overline{0}$. Hence, not every fuzzy approximation separated sets are fuzzy ideal approximation separated sets, and moreover, the fuzzy set $(\mu \vee v)$ will be fuzzy ideal approximation connected set whenever $\ell \neq I^{X}$ and $\ell \neq\{\overline{0}\}$, that is, whenever $\ell$ is a proper fuzzy ideal on $X$.

Proposition 3 Let $(X, R, \ell)$ be a fuzzy ideal approximation space and $\lambda \in I^{X}$. Then, the following are equivalent.

(1) $(X, R, \ell)$ is fuzzy ideal approximation connected.

(2) $\left.\mu \wedge v=\overline{0},\left(\text { int }_{R}^{\lambda}\right)_{\lambda}^{*}(\mu)=\mu,(\text { int })_{R}^{\lambda}\right)_{\lambda}^{*}(v)=v$ and $\mu \vee v=\overline{1} \quad$ imply $\mu=\overline{0}$ or $v=\overline{0}$.

(3) $\mu \wedge v=\overline{0},\left(c l_{R}^{\lambda}\right)_{\lambda}^{*}(\mu)=\mu,\left(c l_{R}^{\lambda}\right)_{\lambda}^{*}(v)=v$ and $\mu \vee v=\overline{1} \quad$ imply $\mu=\overline{0}$ or $v=\overline{0}$.

$\operatorname{Proof}(1) \Rightarrow(2)$ : Let $\mu, v \in I^{X}$ with $\left(\operatorname{int}_{R}^{\lambda}\right)_{\lambda}^{*}(\mu)=\mu,\left(\operatorname{int}_{R}^{\lambda}\right)_{\lambda}^{*}(v)=v$ such that $\mu \wedge v=\overline{0}$ and $\mu \vee v=\overline{1}$. Then, from (2) in Proposition 3.2, we get that

$$
\begin{aligned}
& \left(\operatorname{cl}_{R}^{\lambda}\right)_{\lambda}^{*}(\mu)=\left(\operatorname{cl}_{R}^{\lambda}\right)_{\lambda}^{*}\left(v^{c}\right)=\left(\left(\operatorname{int}_{R}^{\lambda}\right)_{\lambda}^{*}(v)\right)^{c}=v^{c}=\mu, \\
& \left(\operatorname{cl}_{R}^{\lambda}\right)_{\lambda}^{*}(v)=\left(\operatorname{cl}_{R}^{\lambda}\right)_{\lambda}^{*}\left(\mu^{c}\right)=\left(\left(\operatorname{int}_{R}^{\lambda}\right)_{\lambda}^{*}(\mu)\right)^{c}=\mu^{c}=v .
\end{aligned}
$$

Hence, $\left(\operatorname{cl}_{R}^{\lambda}\right)_{\lambda}^{*}(\mu) \wedge v=\mu \wedge\left(\mathrm{cl}_{R}^{\lambda}\right)_{\lambda}^{*}(v)=\mu \wedge v=\overline{0}$. That is, $\mu, v$ are fuzzy ideal approximation separated sets so that $\mu \vee v=\overline{1}$. But $(X, R, \ell)$ is fuzzy ideal approximation connected implies that $\mu=\overline{0}$ or $\nu=\overline{0}$.

(2) $\Rightarrow$ (3): , (3) $\Rightarrow$ (1): Clear. 
Proposition 4 Let $(X, R, \ell)$ be a fuzzy ideal approximation space and $\lambda, \mu \in I^{X}$. Then, the following are equivalent.

(1) $\mu$ is fuzzy ideal approximation connected set.

(2) If $v, \rho$ are fuzzy ideal approximation separated sets with $\mu \leq(v \vee \rho)$, then $\mu \wedge \nu=\overline{0}$ or $\mu \wedge \rho=\overline{0}$.

(3) If $v, \rho$ are fuzzy ideal approximation separated sets with $\mu \leq(v \vee \rho)$, then $\mu \leq v$ or $\mu \leq \rho$.

Proof $(1) \Rightarrow(2)$ : Let $\nu, \rho$ be fuzzy ideal approximation separated sets with $\mu \leq(\nu \vee \rho)$. That is, $\left(\mathrm{cl}_{R}^{\lambda}\right)_{\lambda}^{*}(v) \wedge \rho=\left(\mathrm{cl}_{R}^{\lambda}\right)_{\lambda}^{*}(\rho) \wedge v=\overline{0}$ so that $\mu \leq(v \vee \rho)$. Since

$\left(\mathrm{cl}_{R}^{\lambda}\right)_{\lambda}^{*}(\mu \wedge \nu) \wedge(\mu \wedge \rho)=\left(\mathrm{cl}_{R}^{\lambda}\right)_{\lambda}^{*}(\mu) \wedge\left(\mathrm{cl}_{R}^{\lambda}\right)_{\lambda}^{*}(\nu) \wedge(\mu \wedge \rho)=\left(\mathrm{cl}_{R}^{\lambda}\right)_{\lambda}^{*}(\mu) \wedge \mu \wedge\left(\mathrm{cl}_{R}^{\lambda}\right)_{\lambda}^{*}(\nu) \wedge \rho=\overline{0}$.

$\left(\mathrm{cl}_{R}^{\lambda}\right)_{\lambda}^{*}(\mu \wedge \rho) \wedge(\mu \wedge \nu)=\left(\mathrm{cl}_{R}^{\lambda}\right)_{\lambda}^{*}(\mu) \wedge\left(\mathrm{cl}_{R}^{\lambda}\right)_{\lambda}^{*}(\rho) \wedge(\mu \wedge \nu)=\left(\mathrm{cl}_{R}^{\lambda}\right)_{\lambda}^{*}(\mu) \wedge \mu \wedge\left(\mathrm{cl}_{R}^{\lambda}\right)_{\lambda}^{*}(\rho) \wedge \nu=\overline{0}$.

Then, $(\mu \wedge \nu)$ and $(\mu \wedge \rho)$ are fuzzy ideal approximation separated sets with $\mu=(\mu \wedge \nu) \vee(\mu \wedge \rho)$. But $\mu$ is fuzzy ideal approximation connected means that $\mu \wedge \nu=\overline{0}$ or $\mu \wedge \rho=\overline{0}$.

(2) $\Rightarrow$ (3): If $\mu \wedge v=\overline{0}, \mu \leq(\nu \vee \rho)$ means that $\mu=\mu \wedge(v \vee \rho)=(\mu \wedge \nu) \vee(\mu \wedge \rho)=$ $\mu \wedge \rho$, and thus $\mu \leq \rho$. Also, if $\mu \wedge \rho=\overline{0}$, then $\mu \leq \nu$.

(3) $\Rightarrow$ (1): Let $v, \rho$ be fuzzy ideal approximation separated sets so that $\mu=v \vee \rho$. Then, from (3), $\mu \leq v$ or $\mu \leq \rho$. If $\mu \leq v$, then

$$
\rho=(v \vee \rho) \wedge \rho=\mu \wedge \rho \leq v \wedge \rho \leq\left(\mathrm{cl}_{R}^{\lambda}\right)_{\lambda}^{*}(v) \wedge \rho=\overline{0} .
$$

Also, if $\mu \leq \rho$, then

$$
v=(v \vee \rho) \wedge v=\mu \wedge v \leq \rho \wedge v \leq\left(\mathrm{cl}_{R}^{\lambda}\right)_{\lambda}^{*}(\rho) \wedge v=\overline{0}
$$

Hence, $\mu$ is fuzzy ideal approximation connected set.

Definition 7 Let $(X, R),\left(Y, R^{*}\right)$ be two fuzzy approximation spaces and $\lambda \in I^{X}, \mu \in I^{Y}$ are fuzzy sets. Then, the mapping $f:(X, R) \rightarrow\left(Y, R^{*}\right)$ is called fuzzy approximation continuous (FA-cont.) if

$$
f^{-1}\left(\operatorname{int}_{R^{*}}^{\mu}(v)\right) \leq \operatorname{int} t_{R}^{\lambda}\left(f^{-1}(v)\right) \quad \forall v \in I^{Y} .
$$

Equivalently, fis called fuzzy approximation continuous (FA -cont.) if

$$
f^{-1}\left(c l_{R^{*}}^{\mu}(v)\right) \geq c l_{R}^{\lambda}\left(f^{-1}(v)\right) \quad \forall v \in I^{Y}
$$

Definition $8 \mathrm{~A}$ mapping $f:(X, R, \ell) \rightarrow\left(Y, R^{*}\right)$ is called fuzzy ideal approximation continuous (FIA - cont.) if

$$
f^{-1}\left(\operatorname{int} t_{R^{*}}^{\mu}(v)\right) \leq\left(\operatorname{int} t_{R}^{\lambda}\right)_{\lambda}^{*}\left(f^{-1}(v)\right) \quad \forall v \in I^{Y} .
$$

Equivalently, fis called fuzzy ideal approximation continuous (FIA -cont.) if

$$
f^{-1}\left(c l_{R^{*}}^{\mu}(v)\right) \geq\left(c l_{R}^{\lambda}\right)_{\lambda}^{*}\left(f^{-1}(v)\right) \quad \forall v \in I^{Y} .
$$

Clearly, every fuzzy ideal approximation continuous mapping will be fuzzy approximation continuous as well (from (1) in Proposition 2) but not converse. In the following, we give an example. 
Example 5 Let $X=Y=\{a, b, c, d\}, f: X \rightarrow Y$ a mapping such that $f(a)=f(b)=a, f(c)=b, f(d)=d, R, R^{*}$ are fuzzy equivalence relations on $X, Y$, respectively as follows:

\begin{tabular}{|c||c|c|c|c|}
\hline$R$ & $a$ & $b$ & $c$ & $d$ \\
\hline$a$ & 1 & 0 & 0 & 0 \\
\hline$b$ & 0 & 1 & 0 & 0 \\
\hline$c$ & 0 & 0 & 1 & 0.3 \\
\hline$d$ & 0 & 0 & 0.3 & 1 \\
\hline
\end{tabular}

\begin{tabular}{c||c|c|c|c}
$R^{*}$ & $a$ & $b$ & $c$ & $d$ \\
\hline \hline$a$ & 1 & 0.5 & 0.5 & 0 \\
\hline$b$ & 0.5 & 1 & 0.5 & 0 \\
\hline$c$ & 0.5 & 0.5 & 1 & 0 \\
\hline$d$ & 0 & 0 & 0 & 1 \\
\hline
\end{tabular}

Let $\lambda=\{1,1,1,0.2\} \in I^{X}$ and $\mu=\{0,0.3,0.5,0.2\} \in I^{Y}$ be fixed. Then, $\lambda_{R}=$ $\{1,1,0.7,0.2\},\left(\lambda_{R}\right)^{c}=\{0,0,0.3,0.8\}$ and $\lambda^{R}=\{1,1,1,0.3\}$. For $\eta=\{0,0,0.8,0\} \in I^{Y}$, we get that $f^{-1}(\eta)=\overline{0}$, and then $\mathrm{cl}_{R}^{\lambda}\left(f^{-1}(\eta)\right)=\overline{0}$.

Since $\mu_{R^{*}}=\{0,0.3,0.5,0.2\},\left(\mu_{R^{*}}\right)^{c}=\{1,0.7,0.5,0.8\}$ and $\eta^{R^{*}}=\{0.5,0.5,0,0\}$, then $c l_{R^{*}}^{\mu}(\eta)=\left(\mu_{R^{*}}\right)^{c} \vee \eta^{R^{*}}=\{1,0.7,0.5,0.8\}$. Thus, $f^{-1}\left(\mathrm{cl}_{R^{*}}^{\mu}(\eta)\right)=\{1,1,0.7,0.8\} \geq \overline{0}=$ $\mathrm{cl}_{R}^{\lambda}\left(f^{-1}(\eta)\right)$. Hence, there is a fuzzy set $\eta \in I^{Y}$ satisfying the condition of fuzzy approximation continuity. Next, we will show that $\eta$ itself will not satisfy the condition of fuzzy ideal approximation continuity.

Since, $c l_{R}^{\lambda}\left(f^{-1}(\eta)\right)=\overline{0}$, then $\left(c l_{R}^{\lambda}\right)_{\lambda}^{*}\left(f^{-1}(\eta)\right)=\mathrm{cl}_{R}^{\lambda}\left(f^{-1}(\eta)\right) \vee\left(\lambda^{R}\right)_{\lambda}^{*}=\left(\lambda^{R}\right)_{\lambda}^{*}$, that is, $\left(\mathrm{cl}_{R}^{\lambda}\right)_{\lambda}^{*}\left(f^{-1}(\eta)\right)=\{1,1,1,0.3\}_{\lambda}^{*}$.

Now, define a fuzzy ideal $\ell$ on $X$ as follows, $\xi \in \ell \Longleftrightarrow \xi \leq\{1,1,0.2,0.2\}$. Then, from being $c l_{R}^{\lambda}(\nu)=\left(\lambda_{R}\right)^{c} \vee v^{R} \geq\{0,0,0.3,0.8\} \forall v \in I^{X}$, we get that $\left(\mathrm{cl}_{R}^{\lambda}\right)_{\lambda}^{*}\left(f^{-1}(\eta)\right)=$ $\{1,1,1,0.3\}_{\lambda}^{*}=\{0,0,0.8,0.8\}$ according to the definition of $\ell$ and the definition of $v_{\lambda}^{*}=$ $\bigwedge\left\{\zeta: v \bar{\wedge} \zeta \in \ell, \mathrm{cl}_{R}^{\lambda}(\zeta)=\zeta\right\}$ for any $\nu \in I^{X}$.

Hence, we get that $f^{-1}\left(c l_{R^{*}}^{\mu}(\eta)\right)=\{1,1,0.7,0.8\} \nsupseteq\left(\mathrm{cl}_{R}^{\lambda}\right)_{\lambda}^{*}\left(f^{-1}(\eta)\right)=\{0,0,0.8,0.8\}$, and therefore, not any fuzzy approximation continuous map must be fuzzy ideal approximation continuous but the converse is a must.

Remark 3 Since $\ell$ and $\ell^{*}$ are independent fuzzy ideals on $X$ and $Y$ respectively, then the mapping $f:(X, R, \ell) \rightarrow\left(Y, R^{*}, \ell^{*}\right)$ still not fuzzy ideal approximation continuous in general even if we have taken $f$ is a bijective map with respect to $\lambda \in I^{X}$ and $f(\lambda) \in I^{Y}$ and the fuzzy relations $R$ on $X$ and $R^{*}$ on $Y$ where $R^{*}=R \circ\left(f^{-1} \times f^{-1}\right)=(f \times f)(R)$. This special case itself could be as an example of a fuzzy approximation continuous mapping but not fuzzy ideal approximation continuous in general.

Theorem 1 Let $(X, R, \ell),\left(Y, R^{*}, \ell^{*}\right)$, associated with $\lambda \in I^{X}$ and $\mu \in I^{Y}$ respectively, be fuzzy ideal approximation spaces and $f:(X, R, \ell) \rightarrow\left(Y, R^{*}, \ell^{*}\right)$ is a fuzzy ideal approximation continuous mapping. Then, $f(\eta) \in I^{Y}$ is a fuzzy ideal approximation connected set if $\eta$ is a fuzzy ideal approximation connected set in $X$.

Proof Let $v, \rho \in I^{Y}$ be fuzzy ideal approximation separated sets with $f(\eta)=v \vee \rho$. That is, $\quad\left(\mathrm{cl}_{R^{*}}^{\mu}\right)_{\mu}^{*}(v) \wedge \rho=\left(\mathrm{cl}_{R^{*}}^{\mu}\right)_{\mu}^{*}(\rho) \wedge v=\overline{0}$. Then, $\eta \leq\left(f^{-1}(\nu) \vee f^{-1}(\rho)\right)$, and from $f$ is fuzzy ideal approximation continuous, we get that

$$
\begin{aligned}
\left(\operatorname{cl}_{R}^{\lambda}\right)_{\lambda}^{*}\left(f^{-1}(\nu)\right) \wedge f^{-1}(\rho) & \leq f^{-1}\left(\operatorname{cl}_{R^{*}}^{\mu}(\nu)\right) \wedge f^{-1}(\rho) \\
& =f^{-1}\left(\operatorname{cl}_{R^{*}}^{\mu}(\nu) \wedge \rho\right)=f^{-1}(\overline{0})=\overline{0}
\end{aligned}
$$

and in similar way, we have 


$$
\begin{aligned}
\left(\mathrm{cl}_{R}^{\lambda}\right)_{\lambda}^{*}\left(f^{-1}(\rho)\right) \wedge f^{-1}(v) & \leq f^{-1}\left(\mathrm{cl}_{R^{*}}^{\mu}(\rho)\right) \wedge f^{-1}(\nu) \\
& =f^{-1}\left(\mathrm{cl}_{R^{*}}^{\mu}(\rho) \wedge v\right)=f^{-1}(\overline{0})=\overline{0} .
\end{aligned}
$$

Hence, $f^{-1}(v)$ and $f^{-1}(\rho)$ are fuzzy ideal approximation separated sets in $X$ so that $\eta \leq$ $\left(f^{-1}(\nu) \vee f^{-1}(\rho)\right)$. But from (3) in Proposition 4.2, we get that $\eta \leq f^{-1}(v)$ or $\eta \leq f^{-1}(\rho)$, which means that $f(\eta) \leq v$ or $f(\eta) \leq \rho$. Thus, from that $\eta$ is fuzzy ideal approximation connected set in $X$, and again from (3) in Proposition 4.2, we get that $f(\eta)$ is fuzzy ideal approximation connected in $Y$.

The implications in the following diagram are satisfied whenever $f$ is fuzzy ideal approximation continuous (FIA-cont.).

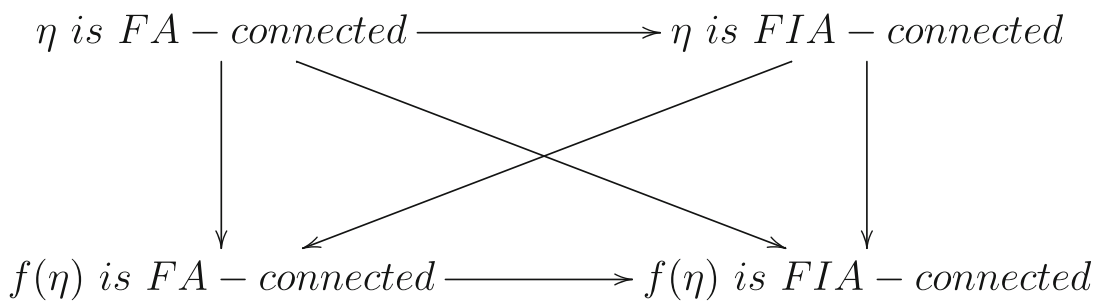

Only the implications in the following diagram are satisfied whenever $f$ is fuzzy approximation continuous (FA - cont.).

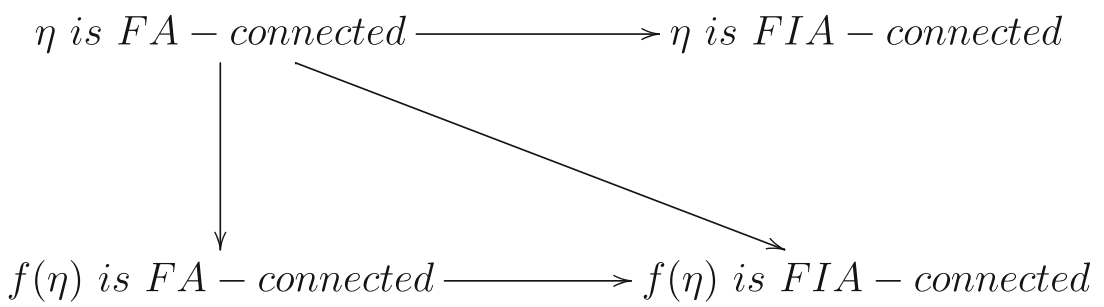

\section{Conclusion}

In this paper, we introduced the fuzzy sets $\lambda_{R}, \lambda^{R}, \lambda^{B}$ for a fuzzy set $\lambda$ that explains the fuzzy roughness of the fuzzy set $\lambda$. We introduced the notion of Fuzzy approximation space and the related fuzzy topology in sense of Chang [14]. Joining a fuzzy ideal to the fuzzy approximation space, we got a fuzzy ideal approximation space with other properties different from those of fuzzy approximation spaces. In a future work, we will define fuzzy approximation rough groups and fuzzy approximation rough rings as applications of this paper.

\section{Acknowledgements}

The authors are thankful to the editor and reviewers for their valuable comments towards improving this paper.

Authors' contributions

The authors contributed equally to this work. The authors read and approved the final manuscript.

Funding

Not applicable.

\section{Availability of data and materials}

Not applicable.

Ethics approval and consent to participate

This article does not contain any studies with human participants or animals performed by any of the authors. 


\section{Consent for publication}

Not applicable.

\section{Competing interests}

The authors declare that they have no competing interests.

\section{Author details}

${ }^{1}$ Department of Mathematics, Faculty of Science, Benha University, Benha, Egypt. ${ }^{2}$ Department of Mathematics, Faculty

of Science, Sohag University, Sohag, Egypt.

Received: 16 August 2019 Accepted: 18 June 2020

Published online: 08 July 2020

\section{References}

1. Pawlak, Z.: Rough sets. Int. J. Inf. Comput. Sci. 11, 341-356 (1982)

2. Zhi, P., Pei, P., Zheng, L.: Topology vs generalized rough sets. Fuzzy Sets Syst. 52(2), 231-239 (2011)

3. Chen, X., Li, Q.: Construction of rough approximations in fuzzy setting. Fuzzy Sets and Syst. 158, 2641-2653 (2007)

4. Dubois, D., Prade, H.: Rough fuzzy sets and fuzzy rough sets. Int. J. Gen. Syst. 17(2-3), 191-209 (1990)

5. Li, Z., Xie, T.: Roughness of fuzzy soft sets and related results. Int. J. Comput. Intell. Syst. 8(2), 278-296 (2015)

6. Liu, G.: Generalized rough sets over fuzzy lattices. Inf. Sci. 178, 1651-1662 (2008)

7. Wu, W-Z, Mi, J-S, Zhang, W-X: Generalized fuzzy rough sets. Inf. Sci. 151, 263-282 (2003)

8. Irfan Ali, M.: A note on soft sets, rough soft sets and fuzzy soft sets. Appl. Soft Comput. 11, 3329-3332 (2011)

9. Molodtsov, D. A.: Soft set theory-first results. Comput. Math. Appl. 37(4-5), 19-31 (1999)

10. Boixader, D., Jacas, J., Recasens, J.: Upper and lower approximations of fuzzy sets. Int. J. of Gen. Sys. 29, 555-568 (2000)

11. Wu, W.-Z:: A study on relationship between fuzzy rough approximation operators and fuzzy topological spaces. Springer-Verlag, Berlin, Heidelberg (2005)

12. Abd El-Latif, A. M.: Generalized soft rough sets and generated soft ideal rough topological spaces. J. Intell. Fuzzy Syst. 34(1), 517-524 (2018)

13. Mahanta, J., Das, P. K.: Fuzzy soft topological spaces. J. Intell. Fuzzy Syst. 32(1), 443-450 (2017)

14. Chang, C. H.: Fuzzy topological spaces. J. of Math. Anal. Appl. 24, 182-190 (1968)

15. Ibedou, I., Abbas, S. E.: Fuzzy topological concepts via ideals and grills. Ann. Fuzzy Math. Inform. 15(2), 137-148 (2018)

16. Sarkar, D.: Fuzzy ideal theory: fuzzy local function and generated fuzzy topology. Fuzzy Sets Syst. 87, 117-123 (1997)

\section{Publisher's Note}

Springer Nature remains neutral with regard to jurisdictional claims in published maps and institutional affiliations.

\section{Submit your manuscript to a SpringerOpen ${ }^{\circ}$ journal and benefit from:}

- Convenient online submission

- Rigorous peer review

- Open access: articles freely available online

- High visibility within the field

Retaining the copyright to your article

Submit your next manuscript at $\gg$ springeropen.com 